\title{
Superimposed disturbance in the ionosphere triggered by spacecraft launches in China
}

\author{
L. M. He ${ }^{1}$ L. X. Wu ${ }^{1,2}$, S. J. Liu ${ }^{1}$ and S. N. Liu ${ }^{1}$ \\ ${ }^{1}$ Institute for Geo-informatics \& Digital Mine Research, College of Resources and Civil Engineering, \\ Northeastern University, Shenyang, 110819, China \\ ${ }^{2}$ School of Environment Science and Spatial Informatics, China University of Mining and Technology, \\ Xuzhou, 221116, China \\ Correspondence to: L. M. He (heliming @ mail.neu.edu.cn)
}

Received: 26 August 2015 - Revised: 22 October 2015 - Accepted: 25 October 2015 - Published: 9 November 2015

\begin{abstract}
Using GPS dual-frequency observations collected by continuously operating GPS tracking stations in China, superimposed disturbances caused by the integrated action of spacecraft's physical effect and chemical effect on ionosphere during the launches of the spacecrafts Tiangong-1 and Shenzhou-8 in China were firstly determined. The results show that the superimposed disturbance was composed of remarkable ionospheric waves and significant ionospheric depletion emerged after both launches. Meanwhile, we found for the first time that the ionospheric waves were made up of two periods of wave by wavelet analysis. The first period of $\sim 4$ min shows one event in the near stations and two subevents in the few far stations. The second period of $\sim 9 \mathrm{~min}$ shows only one event in all the observed stations. Finally, the time characteristics for ionospheric waves and depletions were examined.
\end{abstract}

Keywords. Ionosphere (ionospheric disturbances)

\section{Introduction}

Ionospheric disturbances were observed during spacecraft launches by various traditional ionospheric observation techniques such as ionosonde, incoherent scatter radar, doppler sounder etc. since the early 1960s (Booker, 1961; Felker and Roberts, 1966; Mendillo et al., 1975, 1987; Noble, 1990; Jacobson and Carlos, 1994; Li et al., 1994; Bernhardt et al., 1998, 2005, 2012). Chernogor and Blaunstein (2013) summarized the major characteristic parameters of the observed ionospheric effects on the basis of many space shuttle launch cases processed using incoherent scatter and Doppler radars.
In 1996, Calais and Minster opened new perspectives in ionospheric perturbation detection using global positioning system (GPS) during the launch of STS-58 space shuttle (Calais and Minster, 1996). Afraimovich et al. (2001) used local GPS network to determine the temporal and spatial characteristics of ionospheric disturbances during carrier rocket launches. Recently, Bowling et al. (2013) used an American GPS network to analyze the ionospheric perturbation caused by a space shuttle launch. They further modeled the acoustic wave with periods of 3.3-6.6 min and with the phase speed of $\sim 716 \mathrm{~m} \mathrm{~s}^{-1}$ by ray tracing method. Ding et al. (2014) used a dense GPS network in China to observe the shock and acoustic waves generated during the launch of the Shenzhou10 spacecraft. Long-distance propagation shock and acoustic waves were observed appearing on both sides of the rocket's trajectory with different amplitude of perturbations. These observed disturbances attributed to acoustic waves or shock waves, which are caused by the spacecraft's physical effect characterized by considerably different horizontal velocities and periods.

In addition to the wave disturbances in the ionosphere, another important phenomenon called ionospheric electron depletions or ionospheric holes caused by the gaseous exhaust stream from rocket has been observed by dense GPS network in different regions (Furuya and Heki, 2008; Mendillo et al., 2008; Ozeki and Heki, 2010; Nakashima and Heki, 2014). These observed ionospheric TEC depletions attributed the generation mechanism to the chemical reactions between rocket exhaust gases and charged particles (electrons and ions) of ionosphere, emerging about 6-10 $\mathrm{min}$ after the launches and lasting about $\sim 30-50 \mathrm{~min}$. 
Furthermore, Kakinami et al. (2013) utilized three dense local GPS networks of Japan, Taiwan, and Korea to observe the ionospheric disturbances induced by the Unha-3 launch. A new type of ionospheric disturbance named V-shaped disturbance was observed at a height of $\sim 400 \mathrm{~km}$. The phase velocities of this disturbances were $1.8-2.6 \mathrm{~km} \mathrm{~s}^{-1}$, which is larger than the velocity of acoustic waves reported in previous studies. Lin et al. (2014) observed also V-shaped TEC perturbations with periods of 100-600 s and velocities between $800-1200 \mathrm{~m} \mathrm{~s}^{-1}$ for the 2009 North Korea's Taepodong-2 and 2013 South Korea's Korea Space Launch Vehicle-II rocket launches. These disturbances were attributed to shock waves generated by the rocket's propulsive blasts.

Prior works suggested that the ionospheric perturbations caused by spacecraft launches are complex. On the one hand, most of the previously observed disturbances were interpreted on the basis of a single phenomenon. However, the ionospheric disturbances triggered by spacecraft launch should be an integrated result of spacecraft's physical effect and chemical effect rather than a single effect. In this paper, we first reported the superimposed ionospheric disturbance associated with the integrated action of ionospheric waves caused by the spacecraft's physical effect and ionospheric TEC depletions caused by the spacecraft's chemical effect during the launches of China's spacecrafts Tiangong-1 and Shenzhou-8 in 2011.

On the other hand, the zero-phase filtering method is always used to calculate the waves in ionosphere. Although this method can determine the general behavior of disturbances, it lacks the ability to zoom in and look at some of the finer time-frequency characteristics of the disturbed time series characterized by non-linearity and non-stationarity. Wavelet analysis is a highly effective tool for analyzing nonstationary signals (Mallat, 2008; He et al., 2012). Its property of being localized in time and frequency provides a local time-frequency map of a signal, which can enable the extraction of features varying over time and frequency. For this reason, the ionospheric perturbations produced by the launches of Tiangong-1 and Shenzhou-8 in 2011 are investigated with continuous wavelet transform. More detailed time-frequency characteristics of ionospheric waves with two key disturbed periods caused by the spacecraft's physical effect were revealed firstly by wavelet method in this paper.

\section{Launches of Tiangong-1 and Shenzhou-8}

At 13:16:03 universal time (UT) on 29 September 2011 (day of year, DOY = 272), China's first space lab Tiangong-1 was launched from Jiuquan Satellite Launch Center (JSLC) in Gansu Province by the Long March-2FT1 carrier rocket. According to the China National Space Administration (CNSA), Tiangong-1 is an $8506 \mathrm{~kg}$ space laboratory module, capable of supporting the docking of manned and autonomous spacecraft. One month later, Shenzhou-8, with to- tal mass of $8082 \mathrm{~kg}$, was launched at 21:58:10 UT, 31 October 2011 (DOY = 304), from the same launch center onboard the Changzheng 2F-Y8 carrier rocket to dock with Tiangong-1. Figure 1 shows the observational geometry during the launches of Tiangong-1 and Shenzhou- 8 from JSLC. The Tiangong-1 and Shenzhou- 8 launch track over ground are indicated. It can be seen that the two spacecrafts had almost the same launch orbit.

Two three-station GPS arrays and one IGS station were used to monitor the ionospheric disturbances caused by spacecraft launches in this study. The pink diamonds mark these seven GPS stations in Fig. 1, they were divided into three observation regions including observation region $\mathrm{A}$ (G004, G005, G006), observation region B (G001, G002 G003) and observation region $\mathrm{C}$ (XIAN). The blue and red lines show the trajectories of sub-ionospheric points for GPS satellite 5 and 16 at an altitude of $200 \mathrm{~km}$ for Tiangong- 1 and Shenzhou-8, respectively. Line-of-sight between satellite 5 (PRN05) and the above receivers penetrate the ionosphere near the spacecraft trajectory after the moment of the separation of the first and second stages and before the second stage main engine cutoff and are probably to record the ionospheric disturbance during the Tiangong-1 launch, while satellite 16 (PRN16) is probably to record the ionospheric disturbance during the Shenzhou-8 launch.

\section{GPS-TEC data processing}

The phase and group delays due to the traveling of electromagnetic waves through a dispersive medium can be calculated by the carrier phases and pseudoranges from observations recorded on a dual-frequency GPS receiver. The precise slant total electron content (STEC) integrated along the line of sight (LOS) can be derived from these delays at every observational epoch (Lanyi and Roth, 1988; Mannucci et al., 1998). Besides, the satellite and receiver instrumental biases should be considered in the STEC calculation, and these biases are removed by the ionospheric products from the Center for Orbit Determination in Europe (CODE) and least squares method in this study. We take PRN05 at GPS station G001 as an example. Figure 2a shows the raw STEC time series calculated from GPS dual-frequency observations during 12:30-14:30 UT on DOY 272 in 2011.

Actually, the ionospheric perturbations include acoustic waves and ionospheric depletions that are overlapped on the ionospheric daily variation signals. Therefore, the following data processing strategy was adopted to analyze the ionospheric disturbances caused by spacecraft launch. Firstly, an advanced filtering method (Tarvainen et al., 2002) is initially used to filter the raw STEC time series to separate the high and the low frequency parts of signal by setting a cutoff frequency of $12 \mathrm{~min}$. Figure $2 \mathrm{~b}$ shows the high frequency part of signal, which corresponds to the ionospheric acoustic waves in the signal. Figure $2 \mathrm{c}$ shows the low frequency part of signal, which contains the ionospheric depletion and the iono- 


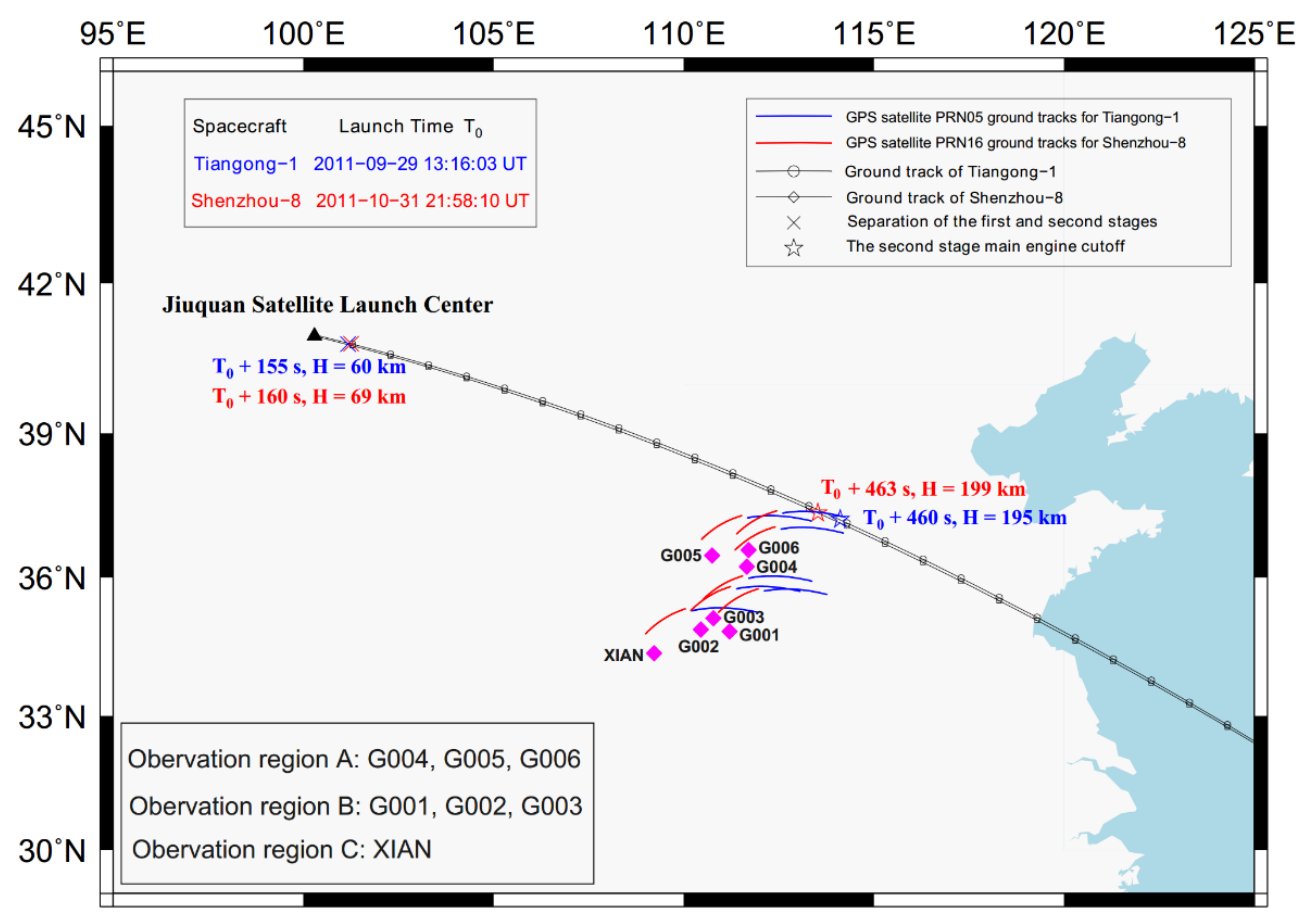

Figure 1. The observational geometry during the launches of Tiangong-1 and Shenzhou- 8 from Jiuquan Satellite Launch Center. The Tiangong-1 and Shenzhou-8 launch track over ground is indicated. The black triangle shows the location of the JSLC. The blue and red crosses represent the locations of Tiangong-1 and Shenzhou- 8 at the moment of the separation of the first and second stages of the rockets, respectively. The blue and red pentacles denote the locations of Tiangong- 1 and Shenzhou- 8 at the moment of the main engine cutoff at the second stage, respectively. The pink diamonds mark seven GPS stations used in this study including observation region A (G004, G005, G006), observation region B (G001, G002 G003) and observation region C (XIAN). The blue and red lines show the trajectories of subionospheric points for GPS satellite 5 and 16 at an altitude of $200 \mathrm{~km}$ for Tiangong- 1 and Shenzhou- 8 , respectively.

spheric daily variation. Then, the least square polynomial fitting was used to calculate the reference curve of STEC (red solid line in Fig. 2c) following Ozeki and Heki (2010) to remove the ionospheric daily variation signal. Here, we used a fourth order polynomials to obtain the ionospheric depletion caused by spacecraft launch (Fig. 2e). Finally, the Morlet wavelet was chosen to perform continuous wavelet transform to further analyze the finer local time-frequency characteristics of the ionospheric waves (Torrence and Compo, 1998; He et al., 2014). Figure $2 \mathrm{~d}$ shows the local wavelet power spectrum of the high frequency part of filtered STEC time series, which indicated two key disturbed periods of wave in the signal.

\section{Ionospheric disturbances}

\subsection{Ionospheric waves caused by Tiangong-1 and Shenzhou-8 launches}

Figure 3a shows the high frequency part of ionospheric STEC for satellite PRN05 of all the seven GPS stations on the day of Tiangong-1 launch (DOY $=272)$ in three observation regions $\mathrm{A}, \mathrm{B}$ and $\mathrm{C}$. It can be seen that clear ionospheric perturbations appeared in all the stations after Tiangong-
1 launch. In order to further identify and describe the localized temporal and periodic variations of the ionospheric perturbations excited by spacecraft launch, the continuous wavelet transformation with Morlet wavelet is applied to examine these time series of ionospheric perturbations in Fig. 3a. Figure 3b, c and d present the local wavelet power spectrum of GPS stations G005, G002 and XIAN for satellites PRN05, respectively. Two significant disturbed periods appeared clearly on the spectrograms several minutes after launching Tiangong- 1 in observation regions $\mathrm{B}$ and $\mathrm{C}$. The period interval of the smaller one is $\sim 2-4 \mathrm{~min}$, while the period interval of the larger one is $\sim 6-12 \mathrm{~min}$. Actually, little disturbance of the small periods also can be seen in observation region $\mathrm{A}$.

There are some typical characteristics of the smaller period disturbance and the larger period disturbance. On the one hand, there exist several same features for the two kinds of disturbance: (1) the amplitude of disturbances increase at first and then decrease with the increase of distance; (2) the largest disturbances occurred in observation region $\mathrm{B}$ for both the two disturbed periods. On the other hand, several different features for the two kinds of disturbances exist: (1) the largest amplitude is different for the two disturbed period, the smaller period disturbance with a larger amplitude 


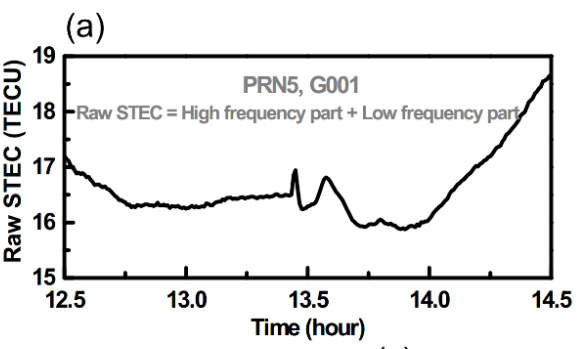

(b)

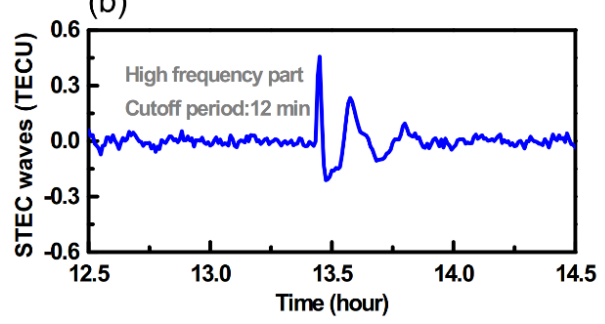

(d)

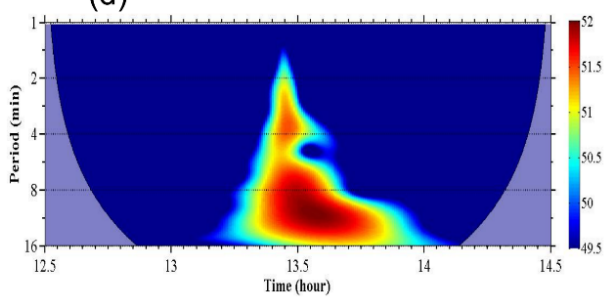

(c)

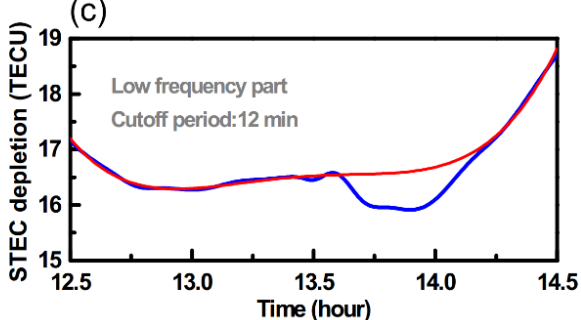

(e)

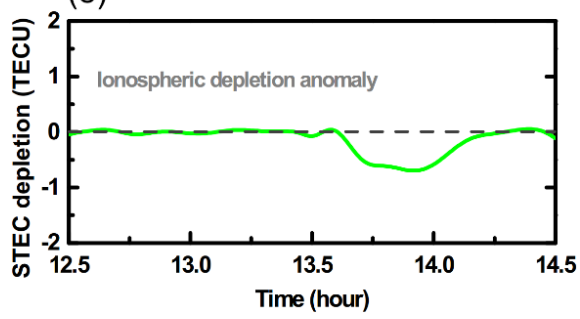

Figure 2. (a) The raw STEC time series of PRN05 at G001 station during 12:30-14:30 UT on DOY 272 in 2011, (b) the high frequency part of filtered STEC time series by setting a cutoff period of $12 \mathrm{~min}$, (c) the low frequency part of filtered STEC time series by setting a cutoff period of $12 \mathrm{~min}$ (blue solid line), the red solid line indicate the reference curve calculated by least squares polynomial fitting method, (d) the local continuous wavelet power spectrum variations of the high frequency part of filtered STEC time series, (e) the ionospheric depletion calculated between the low frequency part of filtered STEC time series and the reference curve.

of $\sim 0.53 \mathrm{TECU}$ while the larger period disturbance with a smaller amplitude of $\sim 0.23$ TECU; (2) it is noteworthy that the smaller period disturbance contains two sub-events in the farthest GPS station XIAN with a distance of $\sim 500 \mathrm{~km}$, while the larger period disturbance shows only one event.

Figure 4 presents similar ionospheric disturbances for satellite PRN16 of all the seven GPS stations on the day of Shenzhou-8 launch (DOY=304). Again, it can be seen that two disturbed periods with strong activity emerged after launching Shenzhou-8. For the higher frequency disturbances in Fig. 4a, c and d, it shows that significant two sub-events fluctuations appeared during the observation period, the first sub-event shows a period interval of 2-4 min. The second sub-event excited by Shenzhou- 8 has a wider period interval of 1-4 min. Aside from the two higher frequency sub-events, a stronger low-frequency perturbation with a period interval of 6-12 min was also observed. Moreover, the amplitude of disturbances increased at first and then decreased with the increase of distance. The largest disturbances occurred in observation region $\mathrm{B}$ for both the two disturbed periods. However, the largest amplitude is different for the two disturbed period, the smaller period disturbance with a larger amplitude of $\sim 0.21$ TECU while the larger pe- riod disturbance with a smaller amplitude of $\sim 0.11$ TECU. The amplitudes of disturbance for the two disturbed periods are smaller than that caused by Tiangong- 1 .

\subsection{Ionospheric depletions caused by Tiangong-1 and Shenzhou-8 launches}

Figure 5a shows the low frequency part of ionospheric STEC for satellite PRN05 of all the seven GPS stations on the day of Tiangong-1 launch (DOY = 272) in the three observation regions. The gray lines in Fig. 5 present reference curves. It can be seen that remarkable ionospheric depletions occurred after launching Tiangong- 1 in all the observations. The amplitude of ionospheric depletion decays with distance from the spacecraft trajectory. The largest depletion occurred in the nearest observation region A with an amplitude up to $\sim 1.4 \mathrm{TECU}$. Figure $5 \mathrm{~b}$ presents the raw STEC and the reference curves with the same time, region and satellite of Fig. 5a. It shows that significant ionospheric disturbances appeared after launching, and these perturbations are likely the products of integrated action of a variety of wave modes and ionospheric depletions. 
(a)

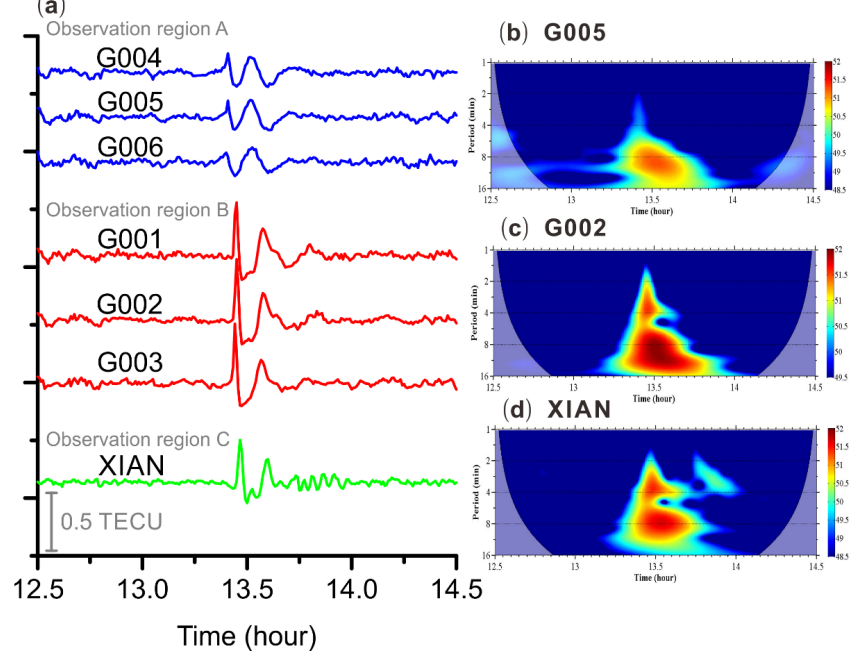

Figure 3. (a) The high frequency part of filtered ionospheric STEC time series for satellite PRN05 of all the seven GPS stations on the day of Tiangong-1 launch $(\mathrm{DOY}=272)$ in three observation regions A, B and C by setting a cutoff period of $12 \mathrm{~min}$; (b) the local wavelet power spectrum of GPS station G005 for satellites PRN05; (c) the local wavelet power spectrum of GPS station G002 for satellites PRN05; (d) the local wavelet power spectrum of GPS station XIAN for satellites PRN05.

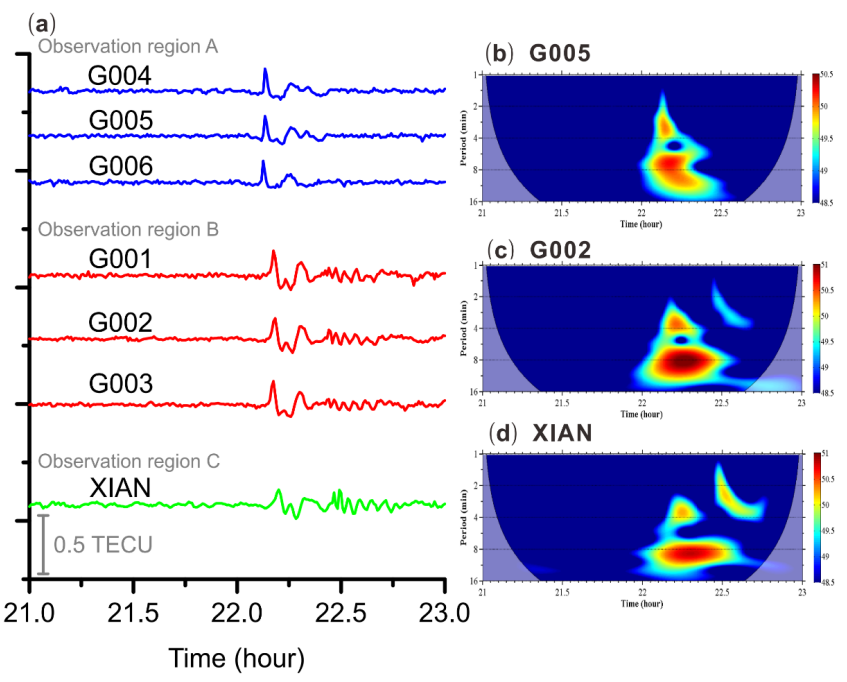

Figure 4. (a) The high frequency part of filtered ionospheric STEC time series for satellite PRN16 of all the seven GPS stations on the day of Shenzhou-8 launch (DOY = 304) in three observation regions $\mathrm{A}, \mathrm{B}$ and $\mathrm{C}$ by setting a cutoff period of $12 \mathrm{~min}$; (b) the local wavelet power spectrum of GPS station G005 for satellites PRN16; (c) the local wavelet power spectrum of GPS station G002 for satellites PRN16; (d) the local wavelet power spectrum of GPS station XIAN for satellites PRN16.

Similarly, Fig. 6a shows the low frequency part of ionospheric STEC for satellite PRN16 of all the seven GPS stations on the day of Shenzhou-8 launch (DOY = 304) in the (a)

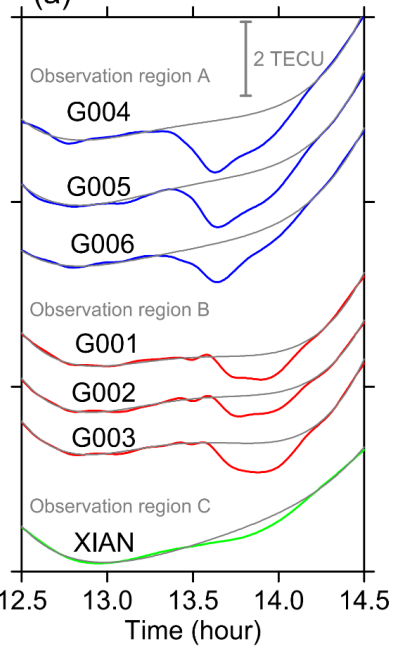

(b)

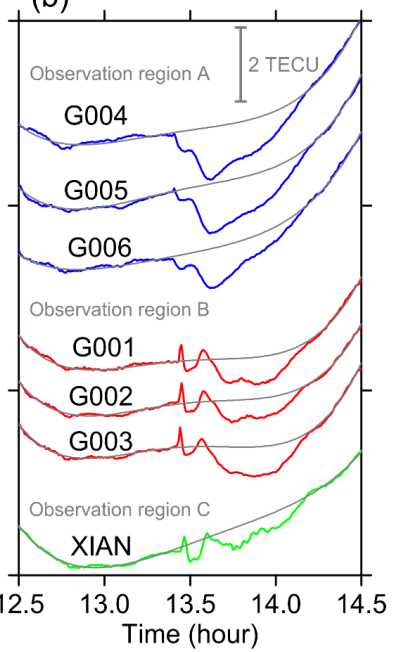

Figure 5. (a) The color curves represent the low frequency part of filtered ionospheric STEC time series for satellite PRN05 of all the seven GPS stations on the day of Tiangong-1 launch $(\mathrm{DOY}=272)$ in three observation regions $\mathrm{A}, \mathrm{B}$ and $\mathrm{C}$ by setting a cutoff period of $12 \mathrm{~min}$, the grey curves represent reference curves calculated by least squares polynomial method for the filtered ionospheric STEC time series; (b) the color curves represent the raw ionospheric STEC time series for satellite PRN05 of all the seven GPS stations on the day of Tiangong-1 launch (DOY = 272) in three observation regions $\mathrm{A}, \mathrm{B}$ and $\mathrm{C}$, the grey curves represent also the reference curves calculated by least squares polynomial method for the filtered ionospheric STEC time series.

three observation regions. The gray lines in Fig. 6 present reference curves. It can be seen that significant ionospheric depletions occurred in observation region $\mathrm{A}$, its amplitude reach up to $\sim 1.97$ TECU. What is different from the ionospheric depletion of Tiangong-1 is that the ionospheric depletion of Shenzhou- 8 does not occur in all the observation regions. The ionospheric depletion was only observed in the nearest observation region. Figure $6 \mathrm{~b}$ presents the raw STEC and the reference curves with the same time, region and satellite of Fig. 6a. It can also be seen that significant ionospheric disturbances appeared after launching, and these perturbations are likely the products of integrated action of a variety of wave modes and ionospheric depletions.

\subsection{Time characteristics of ionospheric disturbances}

To interpret variation features of the appearing time and lasting time for different types of ionospheric disturbance, we take the Tiangong-1 as an example. Figure 7 shows the time characteristics of the ionospheric disturbance variability after launching Tiangong-1. In the observation region A, ionospheric disturbances appeared $\sim 8 \mathrm{~min}$ after the launch and lasted for $\sim 48 \mathrm{~min}$. The integrated action of ionospheric waves and ionospheric depletion emerged after the appearing time and lasted for $\sim 8 \mathrm{~min}$, and the ionospheric deple- 
(a)
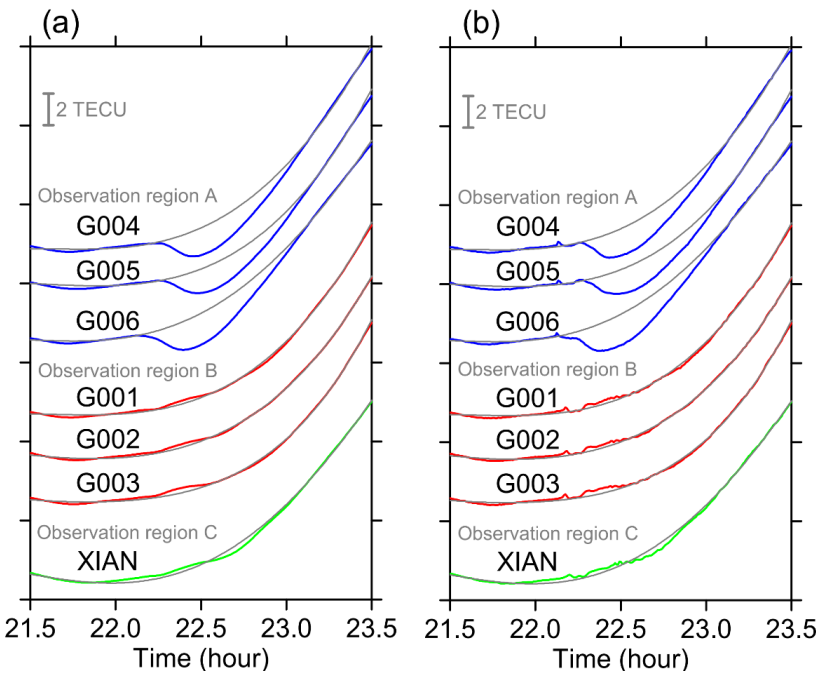

Figure 6. (a) The color curves represent the low frequency part of filtered ionospheric STEC time series for satellite PRN16 of all the seven GPS stations on the day of Shenzhou-8 launch $($ DOY $=304)$ in three observation regions $\mathrm{A}, \mathrm{B}$ and $\mathrm{C}$ by setting a cutoff period of $12 \mathrm{~min}$, the grey curves represent reference curves calculated by least squares polynomial method for the filtered ionospheric STEC time series; (b) the color curves represent the raw ionospheric STEC time series for satellite PRN16 of all the seven GPS stations on the day of Shenzhou-8 launch $(\mathrm{DOY}=304)$ in three observation regions $\mathrm{A}, \mathrm{B}$ and $\mathrm{C}$, the grey curves represent also the reference curves calculated by least squares polynomial method for the filtered ionospheric STEC time series.

tion played the dominant role. Due to the amplitude of ionospheric depletion is much larger than ionospheric wave, it is difficult to find the weak ionospheric waves in the raw STEC time series during the integrated action duration. However, significant ionospheric waves with a period of $\sim 9 \mathrm{~min}$ can be seen in Fig. 3a. In the observation region B, ionospheric disturbances appeared $\sim 10 \mathrm{~min}$ after the launch and lasted for $\sim 45 \mathrm{~min}$, the integrated action of ionospheric waves and ionospheric depletion emerged after the appearing time and lasted for $\sim 12 \mathrm{~min}$, the ionospheric waves played the dominant role. In the observation region $\mathrm{C}$, ionospheric disturbances appeared $\sim 10.5 \mathrm{~min}$ after the launch and lasted for $\sim 43.5 \mathrm{~min}$, the integrated action of ionospheric waves and ionospheric depletion emerged after the appearing time and lasted for $\sim 11 \mathrm{~min}$, the ionospheric waves played the dominant role, and a significant second ionospheric wave overlapped ionospheric depletion for $\sim 15 \mathrm{~min}$.

\section{Discussion and conclusion}

In order to confirm the ionospheric disturbances associated with spacecraft launches, we examined the space environment including solar and geomagnetic activities during the observation periods. For solar activity, the local time of the two launches are at 21:16:03 (Beijing time) and 05:58:10

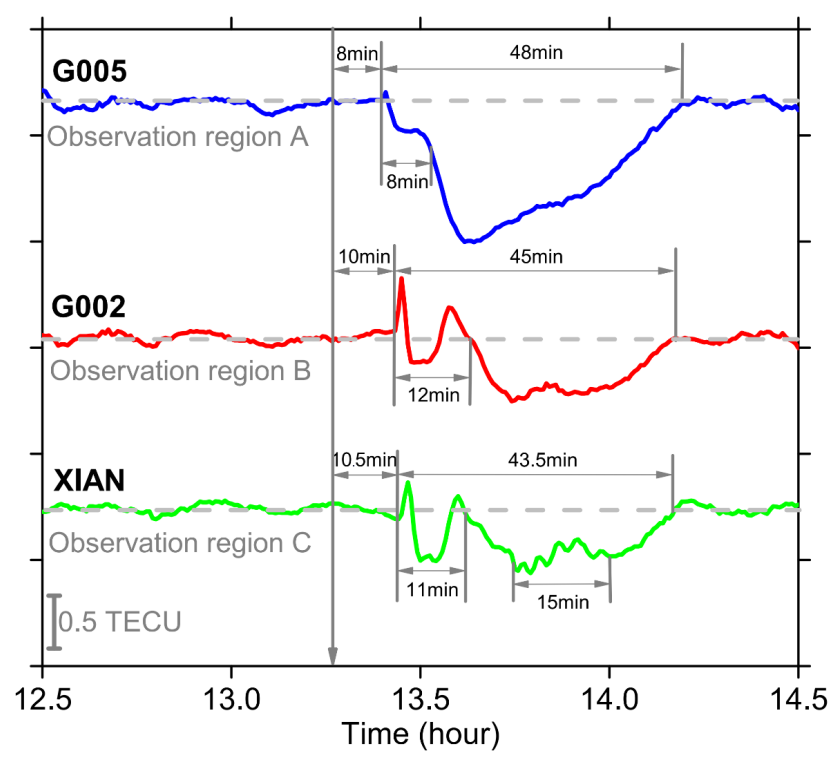

Figure 7. Time characteristics of the ionospheric disturbance variability after launching Tiangong-1 in three observation regions A, $\mathrm{B}$ and $\mathrm{C}$.

(Beijing time), respectively. Therefore, the solar activity has little influence on the ionospheric TEC variation because the two rockets both launched in the night hemisphere. For geomagnetic activity, the 3-h Kp and 1-h Dst values for the launch time of Tiangong- 1 are 3 and $-27 \mathrm{nT}$, and for the launch time of Shenzhou- 8 are 3 and $-6 \mathrm{nT}$, respectively. Besides, we also checked two higher time resolution geomagnetic indices, the USGS 1-min Dst and the 1-min SYM$\mathrm{H}$ (middle latitude symmetric geomagnetic disturbance), and the results confirmed that the geomagnetic environment was relatively quiet during the observation periods.

Furthermore, we also checked the ionospheric variations on the day before and after the Tiangong-1 and Shenzhou8 launches for all the observed satellites. The results show that there was no significant perturbation on the day before and after the two launches. Therefore, the observed complex ionospheric perturbations including multi-wave disturbances with different main frequencies and ionospheric depletions are deemed to be caused by the Tiangong- 1 and Shenzhou- 8 launches.

The mechanism of the observed TEC disturbances induced by spacecraft launches are deduced probably to be twofold: (1) the strong blast wave generated by high-speed rocket, and (2) the chemical gases ejected from rocket exhaust plume. On the one hand, the multi-wave disturbances with complicated frequency and wave structures are products of the physical action between the strong blast wave and the ionospheric electrodynamics process. On the other hand, the ionospheric hole is a product of the chemical reaction between the released gases from rocket and the compositions of ionosphere producing the localized electron density depletion. The ob- 
served results can be explained by the fact that the integrated actions of spacecraft's physical effect and chemical effect on ionosphere during the launches had facilitated the formation of complex ionospheric disturbances.

Overall, the comprehensive disturbed images arose from the spacecraft launches of China's Tianong-1 and Shenzhou8 in 2011 have been detected by ionospheric TEC data. It probably improves our understanding to explain the different results in previous studies. The main results are summarized as follows:

1. The ionospheric disturbances caused by Tiangong-1 and Shenzhou-8 launches are the integrated actions of ionospheric waves and ionospheric depletions. More ionospheric depletions were observed after the launch of Tiangong-1, while more second sub-event waves appeared after the launch of Shenzhou-8. The different characteristics of ionospheric disturbance from the two quite similar launches indicate that the observational geometry plays an important role in the observations and results.

2. The ionospheric waves caused by spacecraft launch contain two key disturbed periods. The first period of $\sim 4$ min showed one event in the near stations and two sub-events in the few far stations. The second period of $\sim 9$ min showed only one event in all the observed stations. The lasting time at different frequencies presented marked variations. These results can explain the different observed frequencies in previous studies due to the single filter method, and it will be helpful to understand the type of waves in the future investigations.

The ionospheric depletion caused by spacecraft launch has tremendous amplitude, which is featured by its large spatial scope and long lasting time. These results have practical significance for local artificial electron depletion experiments, and can lead to a wide range of applications, such as radio communication, GNSS navigation and positioning and so on.

Acknowledgements. This research was supported by the National Important Basic Research Project (973 Program, grant no. 2011CB707102) and the National Natural Science Foundation of China (grant no. 41104104). This study was funded by China Scholarship Council (CSC). The authors thank Angelo De Santis and Sergey Pulinets for their constructive comments and academic suggestions. Figure 1 was produced using the Generic Mapping Tools software (Wessel et al., 1991). The authors acknowledge the International GNSS Service (IGS) and Shanxi administration of surveying, mapping and geoinformation for providing the GPS data. The geomagnetic indices were obtained from World Data Center C2 (WDC-C2) for Geomagnetism in Kyoto. The 1-min Dst indices were downloaded from the USGS.

The topical editor K. Hosokawa thanks A. De Santis and S. Pulinets for help in evaluating this paper.

\section{References}

Afraimovich, E., Kosogorov, E., Perevalova, N., and Plotnikov, A.: The use of GPS arrays in detecting shock-acoustic waves generated during rocket launchings, J. Atmos. Sol.-Terr. Phy., 63, 1941-1957, 2001.

Bernhardt, P., Huba, J., Swartz, W., and Kelley, M.: Incoherent scatter from space shuttle and rocket engine plumes in the ionosphere, J. Geophys. Res., 103, 2239-2251, doi:10.1029/97ja02866, 1998.

Bernhardt, P., Erickson, P., Lind, F., Foster, J., and Reinisch, B.: Artificial disturbances of the ionosphere over the Millstone Hill Incoherent Scatter Radar from dedicated burns of the space shuttle orbital maneuver subsystem engines, J. Geophys. Res., 110, A05311, doi:10.1029/2004ja010795, 2005.

Bernhardt, P., Ballenthin, J., Baumgardner, J., Bhatt, A., Boyd, I., Burt, J., Caton, R., Coster, A., Erickson, P., and Huba, J.: Ground and space-based measurement of rocket engine burns in the ionosphere, IEEE Trans. Plasma Sci., 40, 1267-1286, 2012.

Booker, H. G.: A Local Reduction of F-Region lonization Due to Missile Transit, J. Geophys. Res., 66, 1073-1079, doi:10.1029/JZ066i004p01073, 1961.

Bowling, T., Calais, E., and Haase, J. S.: Detection and modelling of the ionospheric perturbation caused by a Space Shuttle launch using a network of ground-based Global Positioning System stations, Geophys. J. Int., 192, 1324-1331, 2013.

Calais, E. and Minster, J.: GPS detection of ionospheric perturbations following a Space Shuttle ascent, Geophys. Res. Lett., 23, 1897-1900, 1996.

Chernogor, L. F. and Blaunstein, N.: Radiophysical and geomagnetic effects of rocket burn and launch in the near-the-earth environment, Taylor and Francis Group, New York, USA, 534 pp., 2013.

Ding, F., Wan, W., Mao, T., Wang, M., Ning, B., Zhao, B., and Xiong, B.: Ionospheric response to the shock and acoustic waves excited by the launch of the Shenzhou 10 spacecraft, Geophys. Res. Lett., 41, 3351-3358, 2014.

Felker, J. K. and Roberts, W. T.: Ionospheric rarefaction following rocket transit, J. Geophys. Res., 71, 4692-4694, doi:10.1029/JZ071i019p04692, 1966.

Furuya, T. and Heki, K.: Ionospheric hole behind an ascending rocket observed with a dense GPS array, Earth Planets Space, 60, 235-239, 2008.

He, L., Wu, L., Pulinets, S., Liu, S., and Yang, F.: A nonlinear background removal method for seismo-ionospheric anomaly analysis under a complex solar activity scenario: a case study of the M9.0 Tohoku earthquake, Adv. Space Res., 50, 211-220, 2012.

He, L. M., Wu, L. X., De Santis, A., Liu, S. J., and Yang, Y.: Is there a one-to-one correspondence between ionospheric anomalies and large earthquakes along Longmenshan faults?, Ann. Geophys., 32, 187-196, doi:10.5194/angeo-32-187-2014, 2014.

Jacobson, A. R. and Carlos, R. C.: Observations of acoustic-gravity waves in the thermosphere following Space Shuttle ascents, J. Atmos. Terr. Phy., 56, 525-528, 1994.

Kakinami, Y., Yamamoto, M., Chen, C. H., Watanabe, S., Lin, C., Liu, J. Y., and Habu, H.: Ionospheric disturbances induced by a missile launched from North Korea on 12 December 2012, J. Geophys. Res.-Space, 118, 5184-5189, 2013.

Lanyi, G. E. and Roth, T.: A comparison of mapped and measured total ionospheric electron content using global positioning sys- 
tem and beacon satellite observations, Radio Sci., 23, 483-492, doi:10.1029/RS023i004p00483, 1988.

Li, Y., Jacobson, A., Carlos, R., Massey, R., Taranenko, Y., and Wu, G.: The blast wave of the Shuttle plume at ionospheric heights, Geophys. Res. Lett., 21, 2737-2740, 1994.

Lin, C. H., Lin, J. T., Chen, C. H., Liu, J. Y., Sun, Y. Y., Kakinami, Y., Matsumura, M., Chen, W. H., Liu, H., and Rau, R. J.: Ionospheric shock waves triggered by rockets, Ann. Geophys., 32, 1145-1152, doi:10.5194/angeo-32-1145-2014, 2014.

Mallat, S.: A Wavelet Tour of Signal Processing: The Sparse Way, 3rd Edn., Academic Press, New York, USA, 805 pp., 2008.

Mannucci, A., Wilson, B., Yuan, D., Ho, C., Lindqwister, U., and Runge, T.: A global mapping technique for GPS-derived ionospheric total electron content measurements, Radio Sci., 33, 565582, 1998

Mendillo, M., Hawkins, G. S., and Klobuchar, J. A.: A Large-Scale Hole in the Ionosphere Caused by the Launch of Skylab, Science, 187, 343-346, doi:10.1126/science.187.4174.343, 1975.

Mendillo, M., Baumgardner, J., Allen, D. P., Foster, J., Holt, J., Ellis, G. R. A., Klekociuk, A., and Reber, G.: Spacelab-2 Plasma Depletion Experiments for Ionospheric and Radio Astronomical Studies, Science, 238, 1260-1264, doi:10.1126/science.238.4831.1260, 1987.
Mendillo, M., Smith, S., Coster, A., Erickson, P., Baumgardner, J., and Martinis, C.: Man-made space weather, Adv. Space Res., 6, S09001, doi:10.1029/2008SW000406, 2008.

Nakashima, Y. and Heki, K.: Ionospheric hole made by the 2012 North Korean rocket observed with a dense GNSS array in Japan, Radio Sci., 49, 497-505, 2014.

Noble, S.: A large-amplitude traveling ionospheric disturbance excited by the Space Shuttle during launch, J. Geophys. Res., 95, 19037-19044, 1990.

Ozeki, M. and Heki, K.: Ionospheric holes made by ballistic missiles from North Korea detected with a Japanese dense GPS array, J. Geophys. Res., 115, A09314, doi:10.1029/2010JA015531, 2010.

Tarvainen, M. P., Ranta-Aho, P. O., and Karjalainen, P. A.: An advanced detrending method with application to HRV analysis, IEEE Trans. Biomed. Eng., 49, 172-175, 2002.

Torrence, C. and Compo, G.: A Practical Guide to Wavelet Analysis, B. Am. Meteorol. Soc., 79, 61-78, 1998.

Wessel, P. and Smith, W. H. F.: Free software helps map and display data, Trans. Am. Geophys. Union, 72, 441-446, doi:10.1029/90EO00319, 1991. 